\title{
Evaluation of spatial risk factor for leptospirosis outbreak using GIS application
}

\author{
Ahmad Afiq Hassan ${ }^{1}$, Khairul Nizam Tahar 1,2,* \\ ${ }^{1}$ Centre of Studies for Surveying Science and Geomatics, Faculty of Architecture, Planning and Surveying, Universiti Teknologi \\ MARA, 40450 Shah Alam, Selangor, Malaysia \\ ${ }^{2}$ Applied Remote Sensing and Geospatial Research Group, Faculty of Architecture, Planning and Surveying, Universiti Teknologi \\ MARA, 40450 Shah Alam, Selangor, Malaysia
}

\section{A R T I C L E I N F O}

\section{Article history:}

Received 9 May 2016

Received in revised form

25 July 2016

Accepted 26 July 2016

\section{Keywords:}

Spatial analysis

Disease

Prediction

Assessment

Monitoring

\begin{abstract}
A B S T R A C T
The aim of this research is to evaluate the spatial risk factor for high incidence of Leptospirosis outbreak by using GIS application. This research also identifies the factor that increases incidence of Leptospirosis in Petaling district. Data involves in the research is Leptospirosis cases data from Selangor State Health Department Another data is digital base map of Petaling district from Department of Survey and Mapping. The factors used to determine Leptospirosis risk area namely land use, population and temperature data. The land use data is obtained from Town \& Country Planning Department, population data is from Department of Statistics and temperature data is extracted from satellite image using Landsat 8 image. The determination of leptospirosis incidence is made by produce Leptospirosis distribution map (LDM). The LDM is produced using overlay method which is intersecting method and symbology. The identification of factors that increases the leptospirosis incidence is made by producing a Leptospirosis risk map (LRM). A LRM is produced using Getis-OrdGi* technique. The determination of risk area is based on several factors including land use and population factors. The used of GIS application to evaluate the health problem is one of the suitable methods compared with conventional method that made analysis based on the graphs and tables only.

(c) 2016 The Authors. Published by IASE. This is an open access article under the CC BY-NC-ND license (http://creativecommons.org/licenses/by-nc-nd/4.0/).
\end{abstract}

\section{Introduction}

Leptospirosis is an irresistible disease brought on by pathogenic spirochete microorganisms of the sort leptospira that are transmitted straightforwardly or by implication from creatures to human (i.e., a zoonotic disease) and the rodent is the real bearer (host) of the infection (Ministry of Health Malaysia, 2011). Leptospirosis is hazardous on the grounds that it capability to taint a reach creature animal varieties, including people and also the capacity to get by outside the host focused on the natural condition. The fundamental wellsprings of disease are pee of contaminated or transporter creatures (rodent), tainted surface water, mud and soil and the transmission can happen as a consequence of immediate or aberrant contact with the contaminated creature and their emissions

\footnotetext{
* Corresponding Author.

Email Address: nizamtahar@gmail.com (K. N. Tahar)

http://dx.doi.org/10.21833/ijaas.2016.07.010

2313-626X/@ 2016 The Authors. Published by IASE.

This is an open access article under the CC BY-NC-ND license

(http://creativecommons.org/licenses/by-nc-nd/4.0/)
}

(Monahan et al., 2009). Generally, leptospirosis infection shows up in the blood around 4 to 10 hours after contamination and might be noticeable in the blood from a couple of hours to 7 days. Creatures that have recuperated from leptospirosis infection may get to be transporters with the creature exhibit in the renal tubules for times of days to years and will accordingly the infection is shed in the pee into nature.

Fundamentally, irresistible illnesses are transmitted through creature and human developments because of eco-tourism, untamed life research, reintroduction, restoration, chasing, pet exchange, lab, nourishment industry request and cultivating (Beatty et al., 2008). These sorts of developments and exercises are the main consideration that helps for the exchange of leptospirosis to creatures and human and afterward spread the illness to new territories. There are a few conditions that are ideal for transmission of Leptospirosis, for example, supply and bearer have, flooding and waste blockage, creature human interface and human host danger variables (Ministry of Health Malaysia, 2011). For the repository and transporter has, leptospirosis has an extensive 
variety of common rat, and non-rat supply have, for example, rats, dairy cattle, mutts, foxes, rabbits, and so forth. The creatures demonstration as transporters that can discharge an expansive number of leptospirosis in their pee and in charge of the pollution of substantial and little water bodies and soil. Flooding and waste blockage may hazard variables for sullying of water bodies contaminated by creature pee. The other potential for disease builds through recreational exercises without legitimate security. The recreational ranges that poor cleanliness can pull in creature have, for example, rat, hence expands the danger of pollution of the recreational range. These are on the grounds that poor support of offices, dishonourable transfer of waste furthermore open mentality. For the human host danger Figures, a few segments of the populace are more helpless to contamination, for example, those not a while ago laid open to the microorganisms in their surroundings and those with constant sickness and open skin wounds.

Spatial-temporal collaborations among wellbeing occasions are a critical segment in epidemiological and open well-being study, and when managing issues of space, the basic cartography and mapping is a spatial examination, which in geological exploration are the instruments used to analyze the spatial circulation of the gimmicks. In wellbeing research and study, spatial investigation used to discover and evaluate the example of ailment circulation. By spatial investigation can give reasons the spatial example happen, distinguish the area of spatial example happens. Since leptospirosis is a topographical and regular disease controlled by natural and social elements, land data frameworks (GIS) may be valuable to distinguish these components and to focus potential danger territory (Bier et al., 2012). GIS may serve as fundamental for wanting to control and deal with the leptospirosis issue. By utilizing GIS consolidated with spatial examination system permit to mapping the infection, recognizable proof and appraisal of the wellbeing danger variables.

Leptospirosis happens overall and might be a serious issue of health in a damp tropical and subtropical nation such as Malaysia. Despite the fact that leptospirosis cases have been reported in Malaysia since the 1920s, the real sickness trouble in the nation is obscure because of not being a normal disease under the Prevention and Control of Communicable Diseases Act 1988 until now. In Malaysia, an expanding number of reported cases and episodes which had brought about a noteworthy number of passing have been seen over the previous decade. There is an extraordinary requirement for development in case reconnaissance, with a specific end goal to characterize methodologies in control and anticipation of case morbidity and mortality identified with this disease. What is the result of leptospirosis risk map (LRM) using Getis-OrdGi* technique?. This research can help public health agencies to plan the strategies and assist how to control and take preventives activities to control the incidence and also to decrease the incidence cases. Besides that, in Malaysia there is no GIS method has been applied to solve the risk factor for high leptospirosis incidence. This study uses GIS method to evaluate the risk factor that influences the increment of the leptospirosis incidence. The aim of this study is to evaluate the spatial risk factor for high incidence of Leptospirosis outbreak using GIS application. Fig. 1 illustrates the map of Petaling district.

\section{Methodology}

This study involves fourth phases which are planning, data collection, data processing, result and analysis. Phase one discusses planning phase which includes a selection of the research title, selection of study area and hardware and software used. Phase two discusses data collection. In this research, the data that being used to identify Leptospirosis distribution area is Leptospirosis cases data. The data that being used to determine Leptospirosis risk area are population, temperature and land use data. Phase three discusses data processing which includes the production of temperature map, Leptospirosis distribution map and Leptospirosis risk map. These processing have been done using ArcGIS software. Phase four is about result and analysis. In this research, there are two main products that being produce which is Leptospirosis distribution map (LDM) and Leptospirosis risk map (LRM) for the Petaling district. Fig. 2 shows that the research methodology for this research.

In the planning phase, the activities involve are identification the issues of the study and review previous study and article that related to the study of the project. The selection of the title for the study and identification of the location of the study also carried out in this stage including data, hardware and software will be used in the study. The data is collected from Selangor State Health Department and Ministry of Health Malaysia. Another data is a digital map of the study area which is Petaling district that collected from Department of Survey and Mapping or JUPEM. The base map is used as a map to generate the final product of the study area which is Petaling base maps. Furthermore, the factor data that is used to determine Leptospirosis risk area are land use, population and temperature data. Land use data is collected from Town \& Country Planning Department. Population data is collected from Department of Statistics. For the temperature data, the data is used satellite image which is Landsat 8 image. From the image, the temperature data can be extracting using a certain band on the image which is band 10 .

In this research, the software that being used is ArcGIS software as data processing and analysis. The process involves several tools in ArcGIS software that being used to process the data such as overlay, reclassify, symbology, Extract by the mask, raster calculator and Structured Query Language (SQL). The overlay toolset contains tools to overlay 
multiple feature classes to combine, erase, modify or update spatial features that resulting in a new feature class. The new information is created when overlaying one set of features with another. The reclassify tools are providing a variety of method to reclassify or changing the input cell values to alternatives value. There are many reasons for reclassifying the data such as to replace value based on new information, to group certain values together, to reclassify based on the common scale. Symbolization is the process of choosing how to represent the features on a map.

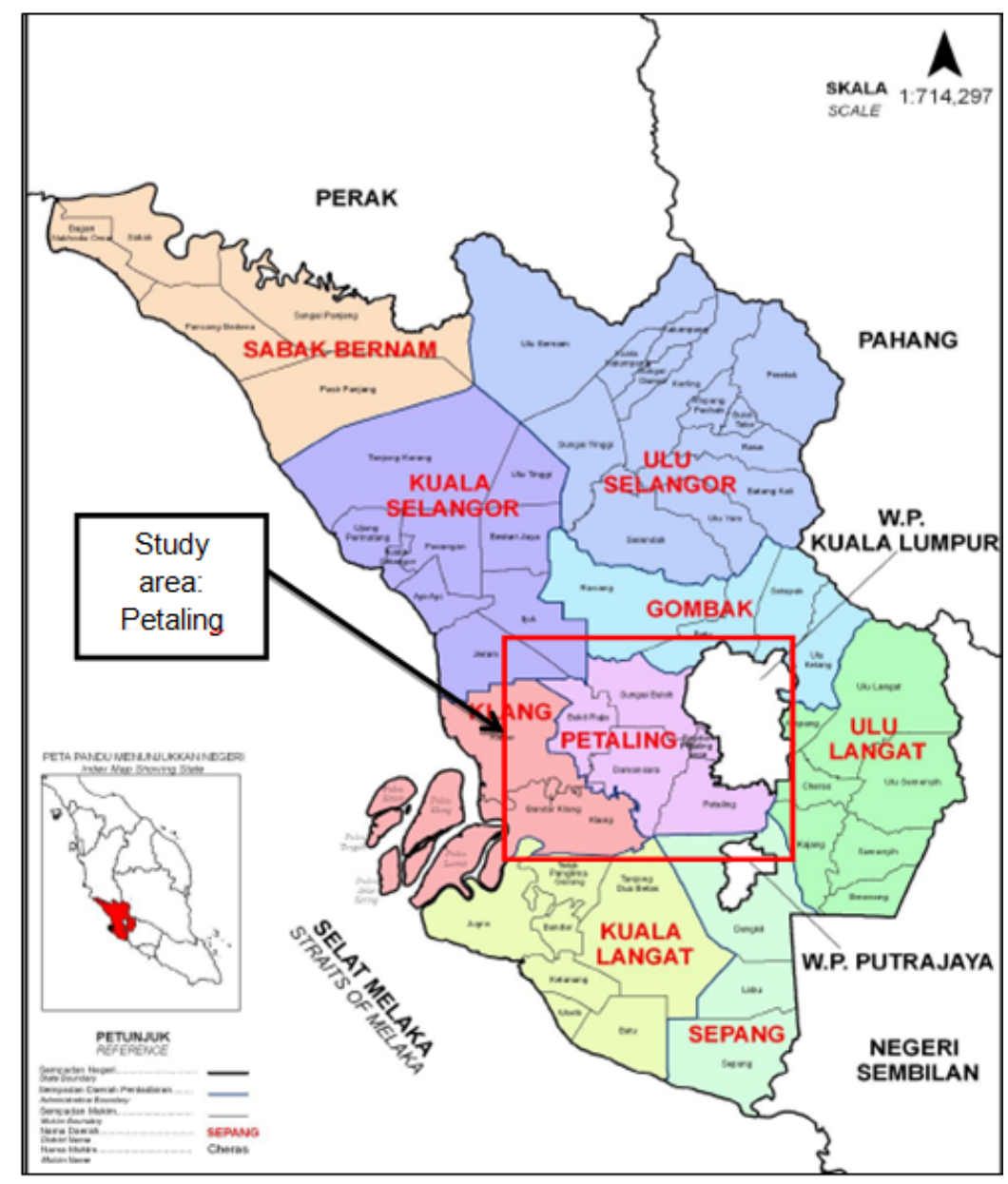

Fig. 1: Study area

The symbols that are chosen should help to describe additional information about the features on the map. Symbolization is an important process because poor symbolization will lead to inaccurate, misunderstood or even deceptive information while effective or better symbolization will help to communicate and describe information quickly and clearly to users or audience. Extract by the mask is a toolset in spatial analyst tool that uses to extracts the cells of a raster that correspond to the areas defined by a mask. The tools are similar to setting the mask environment except that the input mask is only used in the immediate instance while a mask set in the environment is applied to all tools until it is changed or disabled.

\section{Results and analysis}

\subsection{Temperature map using landsat 8 satellite image}

By using Landsat 8 satellite image, the extracted of the temperature data from the satellite image involve several steps and process in ArcGIS software. The first step is selecting temperature band in the Landsat 8 image. Basically, when the data being download on the website, the raw data will have many bands. For the Landsat 8 image, there are 11 bands and the temperature band is either band 10 or band 11 . Select the band 10 and add the band 10 image into ArcGIS. Band 10 image is being used to extract the temperature data in Landsat 8 satellite image.

Then, the second step is to select the study area on the satellite image by using mask tool in ArcGIS. Basically, mask tool needs the reference features as a guide or reference to select the study area. For the task, the Petaling district features can be used as a reference feature to select Petaling area on the satellite image.

Then, the next step is using the raster calculator tool to calculate the digital value in the image to Celsius value by using formula. There are three formulas that being use to get the Celsius value. The first formula is used to convert from operational land imager (OLI) and Thermal Infrared sensor 
(TIRS) band data to Top of Atmosphere (TOA) spectral radiance (Eq. 1)

$$
\mathrm{L} \lambda=\mathrm{MLQcal}+\mathrm{AL}
$$

where:

$\mathrm{L} \lambda=\mathrm{TOA}$ spectral radiance

$\mathrm{ML}=$ Band-specified multiplicative rescaling factor from the metadata

$\mathrm{AL}=$ Band-specified additive rescaling factor from the metadata

Qcal $=$ Quantized and calibrated standard product pixel values (DN)

The second formula is used to convert from spectral radiance to brightness temperature or kelvin by using the thermal constants provided in the metadata file (Eq. 2)

$$
T=\frac{K_{2}}{\ln \left(\frac{K_{1}}{L_{\lambda}}+1\right)}
$$

where:

$T=$ At-satellite brightness temperature $(\mathrm{K})$

$L_{\lambda}=$ TOA spectral radiance

$K_{1}=$ Band-specified thermal conversion constant from the metadata

$K_{2}=$ Band-specified thermal conversion constant from the metadata

Then, the next conversion is from kelvin value to Celsius value by minus kelvin value with the constant value of 273.15 (Eq. 3). Fig. 3 shows the temperature area of Petaling area in Celsius.

$$
\mathrm{T}-273.15
$$

where:

$T=$ At-satellite brightness temperature $(\mathrm{K})$

$273.15=$ constant value to convert from kelvin value to Celsius value.

Then, the next step is to display the image by symbology the image. The temperature area is use as one of the factor to determine risk area for Leptospirosis incidence in Petaling. Then, create temperature station on every area of Petaling district. The temperature station is sample point that has the temperature value that used to estimate the neighbourhood temperature value by using the kriging technique. The temperature value of the sample point is referring the temperature data from Landsat image. Kriging will assume the distances or direction between sample points reflect a spatial correlation that can be used to explain variation in the surface. This technique fits a mathematical function to determine the output value for each location.

\subsection{Leptospirosis distribution area}

In this research, the flow chart of processing is divided into three parts which are to find the Leptospirosis distribution area, to predict the Leptospirosis risk area and to create the temperature map. There are two layers required to determine Leptospirosis distribution area namely Selangor base map and Leptospirosis cases. The first step is selecting the study area of the research which is Petaling district. The method to select study area on Selangor state base map is by using SQL technique (select by attributes). Then, export the selecting area and save it in shape file format. Then, the next step is overlain the two layers which are Leptospirosis cases and Petaling district. The overlay technique that being used is intersected method that computes a geometric intersection of the input features and feature which overlap in all layers will be written to the output features class. By using analysis tools in arc toolbox, choose to intersect in overlay tool, intersect these layers. Then, from the intersect process, the new layer is created. Then, the leptospirosis distribution map is start by uses symbology and classifies the leptospirosis cases into five classes.

\subsection{Leptospirosis risk area}

The next processing is predicted the Leptospirosis risk area in Petaling district. In the process, there are two layers that being used which are Population and Land use layer. The process to get the leptospirosis risk area begins by overlay the two layer using intersect technique. The population layer is intersected with land use layer. By using analysis tools in arc toolbox, select intersect tool in overlay toolset and select two layers which are population and land use and intersect the data. After complete of the intersecting process, the new layer will be created that contain all the data from these different layers. Table 1 shows the weighted value that given to the land use and population data and this weighted value will be used to predict the risk area in Petaling district.

Each of land use and population layers has its own weighted value. The value is based on the previous study and another journal that state the criteria for risk area and their risk level. Furthermore, the guideline from Department of Public Health, Malaysia also stated the high groups to get the Leptospirosis disease such as workers in the agricultural sectors, people involve with recreational activities such as water recreational activities and jungle trekking and travellers or participants in jungle adventure trips or outdoor spot activities. The process assigned weighted value begins by adding a new field at intersect layer and give the name of the field as a weight value. Then by using the SQL (select by attribute) technique, query the classification of land use and added the new information by using field calculator. Then, add new information to all categories of land use based on the weighted value.

The next step predicts the risk area by using spatial statistical tools in arc toolbox. Statistics analysis can help to extract additional information from GIS. In this research, the tools that being use to predict the risk area is using Getis-Ord Gi* tools under mapping cluster toolset in spatial statistic tool. 
The mapping cluster will perform cluster analysis to identify the location of statistically significant hot spot, cold spot, and spatial outlier. The Getis-Ord Gi* tool will calculate the Getis-Ord Gi* statistic for each feature in a dataset. The resultant z-scores and pvalues will tell where features with either high or low values cluster spatially. The prediction of risk area begins by using Getis-Ord $\mathrm{Gi}^{*}$ tool. On the GetisOrd $\mathrm{Gi}^{*}$ box, enter all the suitable information and save the output features. The output features of the Getis-Ord $\mathrm{Gi}^{*}$ process that show in the $\mathrm{Z}$ score. The range of standard deviation value is differing using different colour and value.

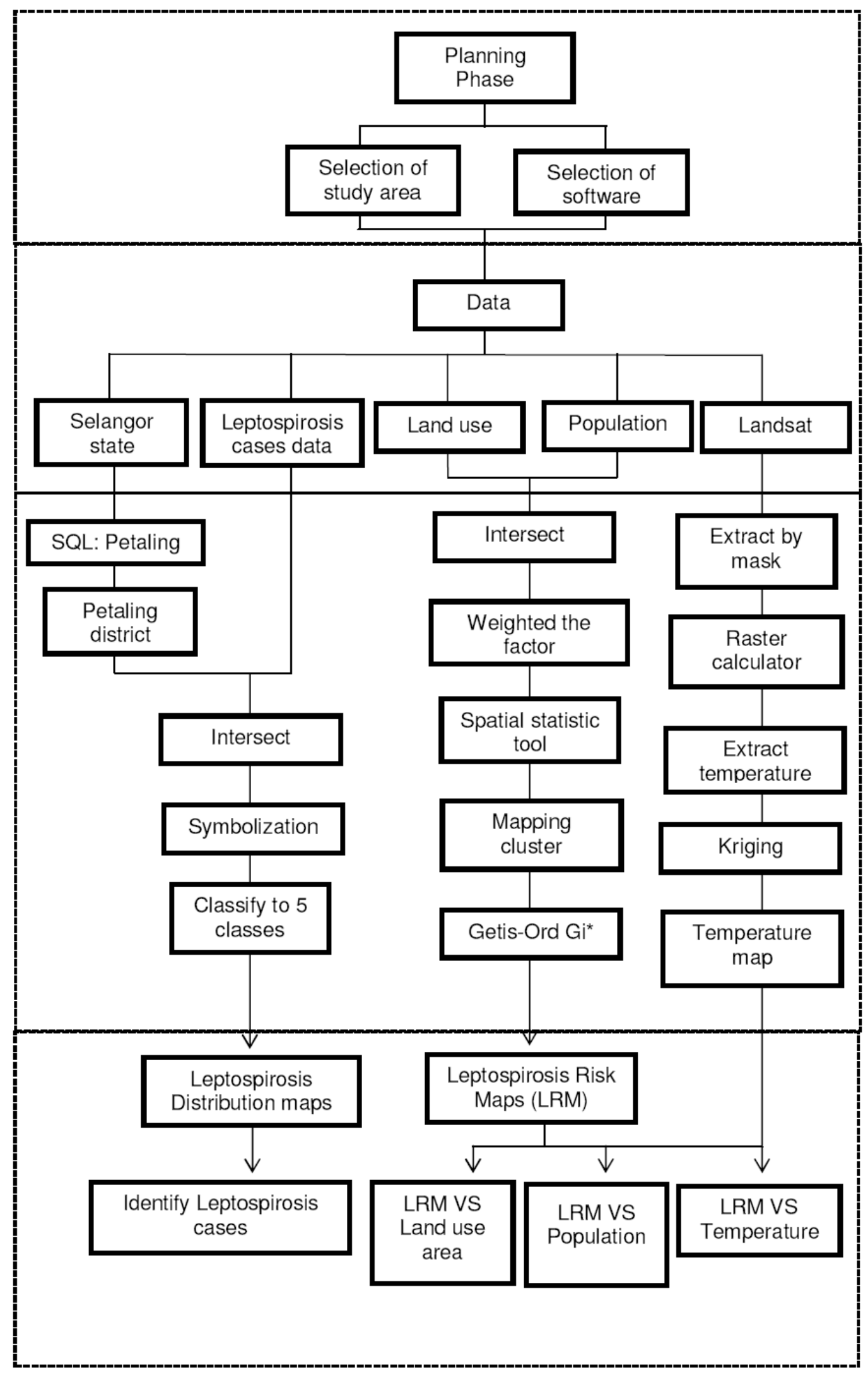

Fig. 2: Research methodology 


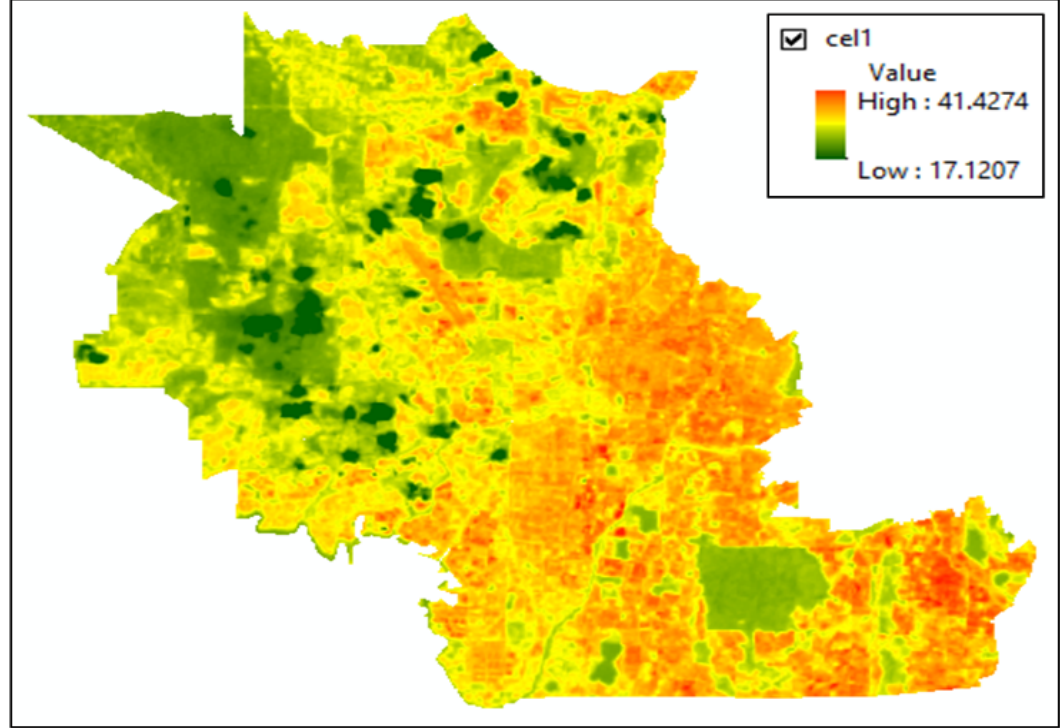

Fig. 3: Temperature area in Celsius

Table 1: The weighted value

\begin{tabular}{|c|c|c|}
\hline Data & Categorize & Weight value \\
\hline & Agriculture area & 5.0 \\
& Forestry area & 5.0 \\
& River area & 5.0 \\
& Recreational area & 5.0 \\
& Industrial area & 3.0 \\
& Residential area & 3.0 \\
& Road area & 1.0 \\
& Utility area & 1.0 \\
\hline Population & More than 100,000 & 5.0 \\
\hline
\end{tabular}

The next process is selecting the risk area level either the area is a high-risk area, moderate risk area or low-risk area. The selecting of the risk area level is by classifying the output layer with three class level which is low risk, moderate risk and high risk; the ranges value based on the value of Z-score of prediction area. Fig. 4 shows the risk area and their rating of risk areas such as high-risk area, moderate risk area and low-risk area. The figure shows that the rating of risk area is distinguished by three different colour displays. The red colour shows the high-risk area, the yellow colour shows the moderate risk area and the green colour show the low-risk area. Then, the last step is to making the map for the Leptospirosis risk area as the final product for the research.

\section{Discussions}

\subsection{Temperature area vs Leptospirosis risks area}

The previous study stated that the potential of Leptospirosis occurs in epidemics in both tropical and temperate climes. The transmission of Leptospirosis can occur world-wide expect the areas with enduring snow or ice and desert, but the most common environment in the tropics and can occur either in urban or rural environments of both industrialized and developing countries.

Fig. 5 shows the comparison between temperature area and Leptospirosis risk area. When the comparison is made between temperature area and Leptospirosis risk area, the result found that the temperature at the high-risk area is around 20 Celsius to 30 Celsius. The result explains that the leptospirosis virus can be survived at mild temperature. It is shown that the potential for Leptospirosis incidence will happen at mild temperature is high compare to the hot temperature.

\subsection{Land use area vs Leptospirosis risk area}

Based on the guidelines for the diagnosis, management, prevention and control of Leptospirosis in Malaysia from Department of Public Health, Malaysia stated that some groups are at higher risk to get the disease such as workers in the agricultural sector, people involves with outdoor or recreational activities such as water recreational activities and jungle trekking, travellers or participants in jungle adventure trips or outdoor sport activities.

The comparison is made between land use area and leptospirosis risk area. The comparison is made for the purposes to know the effect of land use area in leptospirosis incidence and also to identify the level of risk area is located at categories land use. It shows that the high-risk area is located at agriculture area, forestry area and recreational area. The moderate risk area is located at industrial area and for the low-risk area is located in a residential area. The figure also shows that the agriculture area, forestry area and recreational area is contributed to 
the high-risk area for the leptospirosis incidence and the people that involved in that area especially at recreational area or work on that area including agriculture area and forestry area have high potential to get leptospirosis disease if the virus of the disease has on the place.

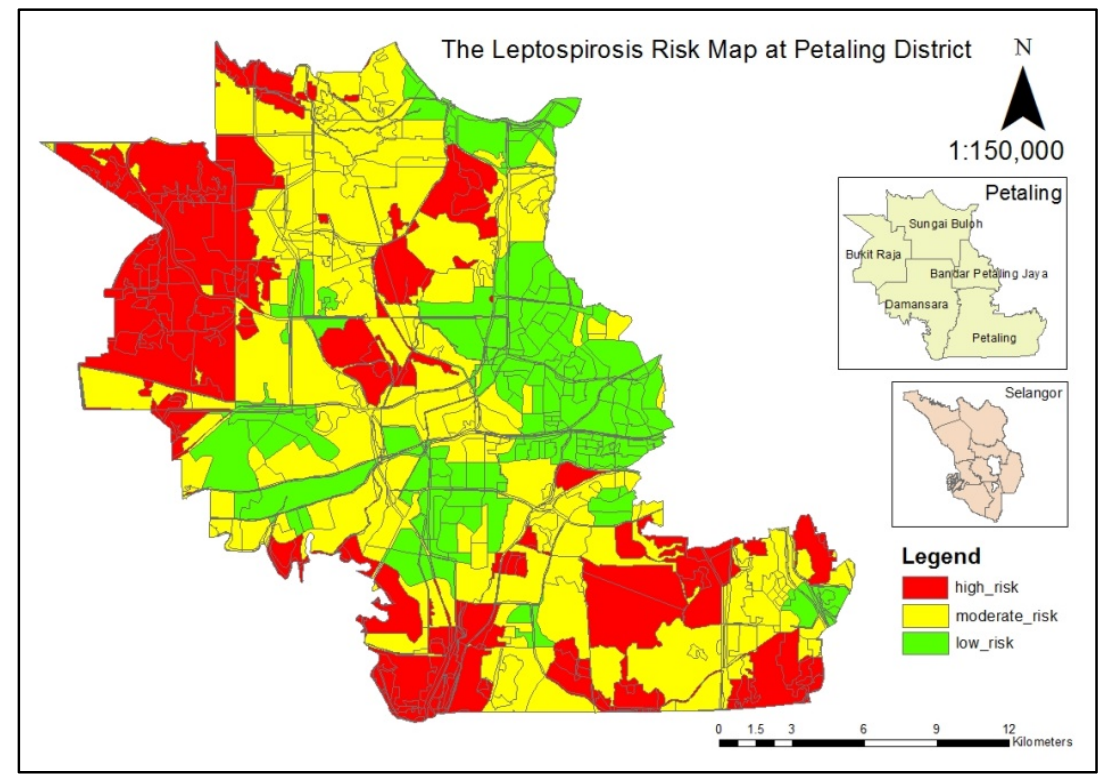

Fig. 4: Leptospirosis Risk Maps
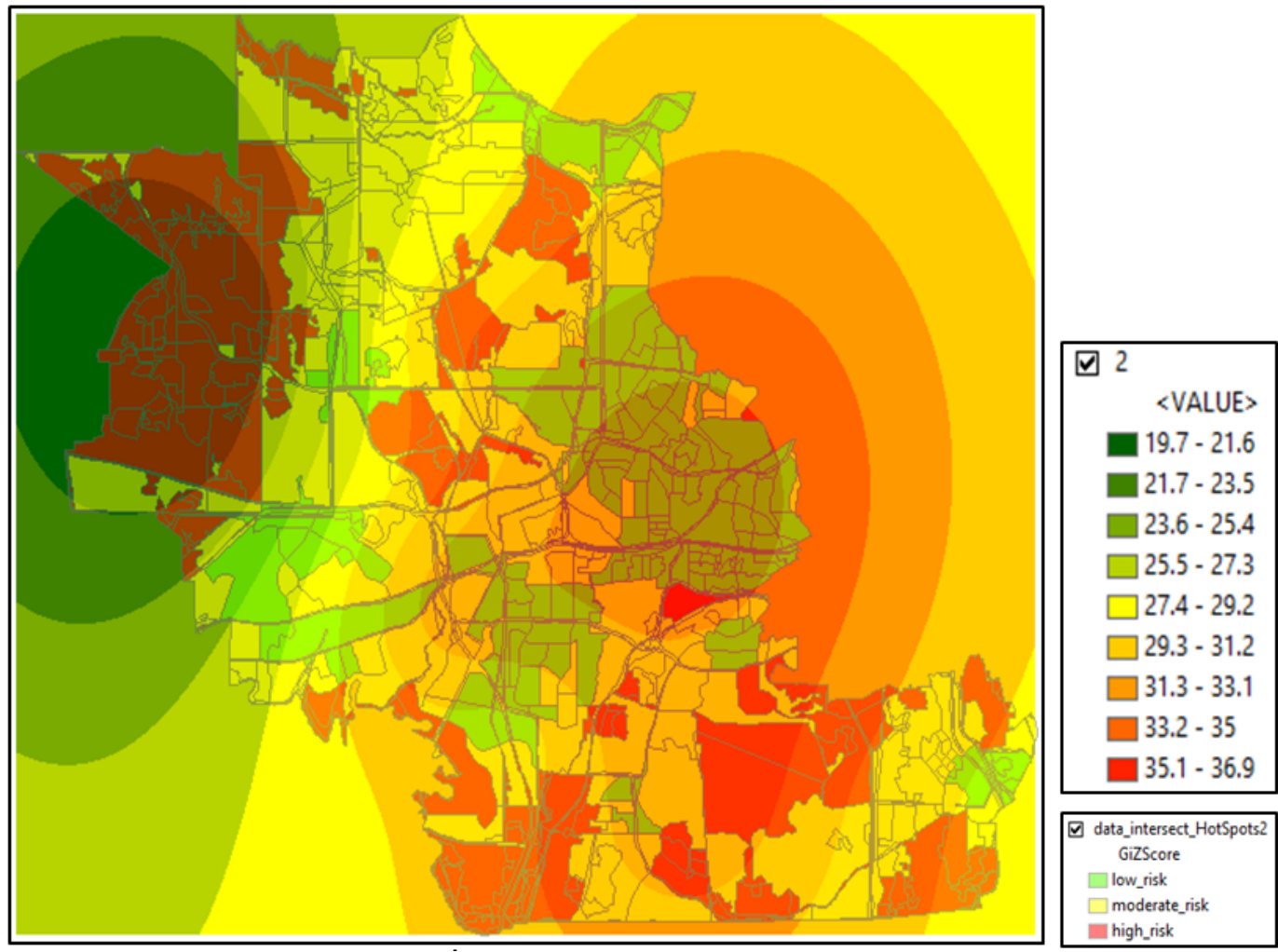

Fig. 5: Temperature area versus Leptospirosis risk area

\subsection{High-density population vs Leptospirosis risk area}

Based on the previous research mentions that the high risk for Leptospirosis incidence will happen is at high-density population either at the town, at the village or at development location. Fig. 6 shows the comparison is made between three ranges of the population

Therefore, there is three risk area is selected within three range of population which are 100,000,
300,000 and 500,000 . The figure shows that the higher population density is one of the risk factor for Leptospirosis incidence will happen. Table 2 shows the total percentage of the area of risk level which is a high-risk area, moderate risk area and low-risk area on three different population ranges.

The 100,000 population density shows that the percentage of high-risk area is $30 \%$, the percentage of moderate risk area is $30 \%$ and the percentage of low-risk area is $40 \%$. The 300,000 population density shows that the percentage of high-risk area 
is $40 \%$, the percentage of moderate risk area is $50 \%$ and the percentage of low-risk area is $10 \%$. The 500,000 population density shows that the percentage of high-risk area is $50 \%$, the percentage of moderate risk area is $40 \%$ and the percentage of low-risk area is $10 \%$. Based on the result that shows in the table above, it can conclude that the higher population density is one of the factors that contribute the Leptospirosis incidence and also the higher population density at that location, the higher potential for Leptospirosis incidence will happen on that location.

Table 2: The percentage of risk level versus population ranges

\begin{tabular}{|c|c|c|c|}
\hline Population ranges & High-risk area (\%) & Moderate risk area (\%) & Low-risk area (\%) \\
\hline 100,000 & 30 & 30 & 40 \\
\hline 300,000 & 40 & 50 & 10 \\
\hline 500,000 & 50 & 40 & 10 \\
\hline
\end{tabular}
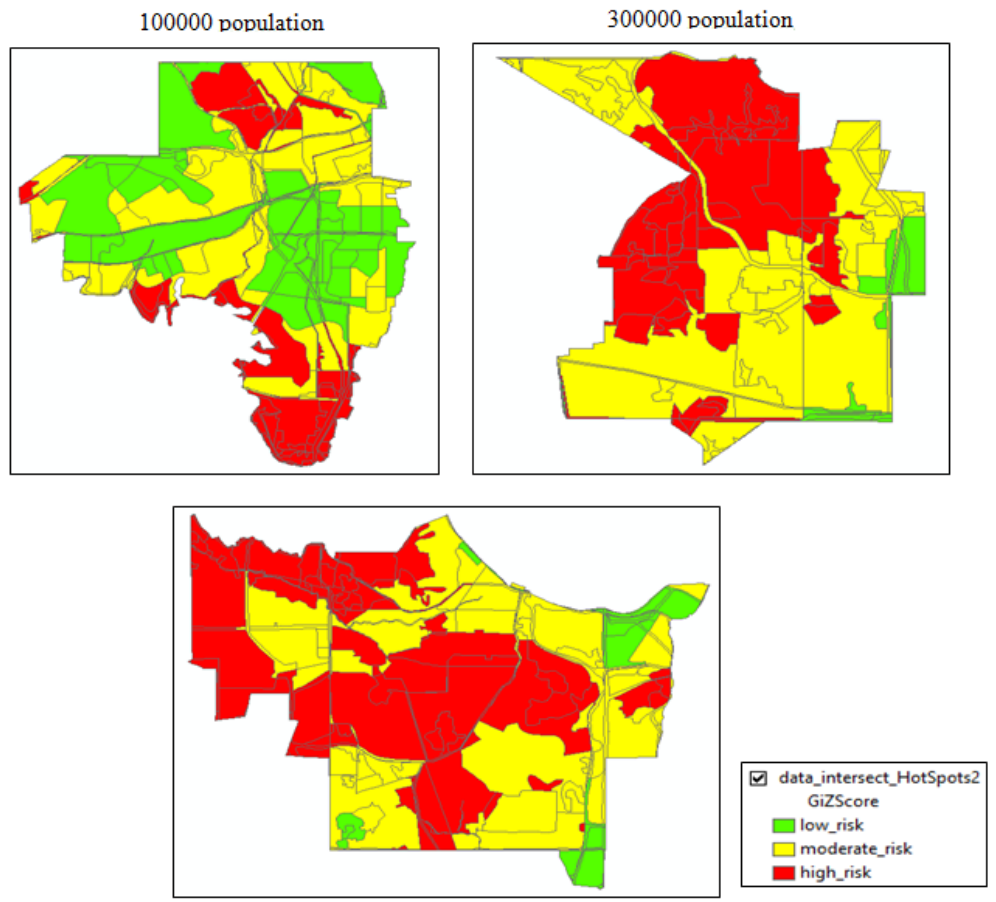

500000 population

Fig. 6: High-density population versus risk area

\section{Conclusions and recommendations}

In this research, the aim is to evaluate the spatial risk factor for high incidence of Leptospirosis outbreak using GIS application. The spatial risk factors that being used are temperature data, land use data and also population data; based on result show that environmental, occupational and recreational is shown the high contribution to Leptospirosis incidence. There are many of articles and books that describe the role and significance of these factors in the tropical climate. The objectives to support the aim is first to determine the incidence of Leptospirosis in the study area. In this research, the determination of Leptospirosis incidence is made by produce Leptospirosis distribution map (LDM). The map shows the location of incidence that happens in the study area and also the analysis can be made based on the LDM map. The second objective is to identify the factor that increases the incidence of Leptospirosis in the study area. Based on result and evaluation, it shows that there is a certain factor that can contribute the increasing of Leptospirosis incidences such as land use, temperature and population. For the land use, the places such as agriculture area, recreational area and forestry area have contributed the increasing of Leptospirosis incidence. For the temperature, the temperature ranges around 20 Celsius to 30 Celsius contributed the increasing of Leptospirosis incidence. For the population, the high-density population contributed the increasing of Leptospirosis incidence. The third objective is to produce a Leptospirosis risk map (LRM) that can be used to control and prevent the increasing of Leptospirosis incidence in the study area. Furthermore, the used of GIS application to evaluate the health problem is one of the suitable methods compared with a conventional method that made an analysis based on the graphs and tables only. GIS has an advantage such as can facilitate a disease data that can be accessed quickly even in large numbers, providing a dynamic system analysis and has a good technique to display, monitor and manage disease outbreak well.

Based on the research conducted, there is several suggestion or recommendations which may beneficial for the further research in the future. The first recommendation is the study area of the research can be expanded to all district in Selangor 
state and the whole of Peninsular Malaysia state. The second recommendation is the use of GIS with integration with remote sensing can also be used as another method or technique to find the Leptospirosis risk area. The third recommendation is used additional factors such as rainfall factor and flood area factor to make the prediction of risk area is more accurate. For the fourth recommendation is using another technique to predict the risk area. For this research, the technique to predict the Leptospirosis risk area is using Getis-Ord Gi* which is applying in ArcGIS software. For the further future study, there are several techniques can be used to predict the risk area including regression analysis technique such as Geographically weighted regression (GWR) or Ordinary least squares (OLS) and also kernel density estimation technique.

\section{Acknowledgements}

Faculty of Architecture, Planning and Surveying Universiti Teknologi MARA (UiTM), Research Management Institute (RMi) and Ministry of Higher Education (MOHE) are greatly acknowledged because providing the fund RAGS 600-RMI/RAGS 5/3 (241/2014), RAGS/1/2014/TK09/UITM/3 to enable this study is carried out.

\section{References}

Beatty A, Scott K and Tsai P (2008). Achieving sustainable global capacity for surveillance and response to emerging diseases of zoonotic origin: workshop summary. National Academies Press, Washington, DC, USA.

Bier D, Martins-Bedê F T, Morikawa VM, Ullmann LS, Kikuti M, Langoni H, \& Molento MB (2012). Spatial Distribution of Seropositive Dogs to Leptospira spp and evaluation of leptospirosis risk factors using a decision tree. Acta Scientiae Veterinariae, 40(3): 1-7.

Ministry of Health Malaysia (2011). Guidelines for the diagnosis, management, prevention and control of Leptospirosis in Malaysia. 1st Edition, Disease Control Division, Department of Public Health, Kuala Lumpur, Malaysia.

Monahan AM, Miller IS and Nally JE (2009). Leptospirosis: risks during recreational activities. Journal of Applied Microbiology, 107(3): 707716. 